\title{
Differential effects of two types of aversive arousal on discriminability'
}

JAMES BIERI, University of Texas, Austin, Tex. 78712, and BRUCE MEYERS, Brooklyn College, Brooklyn, N.Y. 11210

The effects of two types of aversive arousal, white noise and electric shock, upon discriminability was investigated in two studies using information analysis. Results indicated that while discriminability was affected in both studies as a function of arousal, there was a differential effect in the unreliability. component of $S s^{\prime}$ responses. White noise increased the unreliability of responding while shock tended to decrease the unreliability. These differences are discussed in terms of drive and attention properties of the two forms of arousal.

It is apparent from the recent critical review of Spence \& Spence (1966) that various types of arousal may have differing effects on learning and that there is need for more careful empirical and conceptual analysis of this problem. Certainly in relation to discrimination behavior, this same concern would be germane. Two common forms of aversive arousal are electric shock and white noise, but seldom has the comparability of the effects of both been studied in the same design. To analyze the effects of arousal upon discriminability it is useful to study component processes in discrimination by means of information analysis (Garner, 1962; Bieri, Atkins, Briar, Leaman, Miller, \& Tripodi, 1966). An overall index of discriminability can be obtained by use of the measure Txy, or amount of information transmitted reliably. In addition, components of this Txy measure can be analyzed, including the amount of information or variability in the responses of $S$, $\mathrm{Hy}$, and the amount of unreliability in S's responses, Hxy. Overall accuracy of discrimination is enhanced by increases in $\mathrm{Hy}$ and decreases in $\mathrm{Hxy}$, and it is reasonable to assume that increased arousal might affect either or both of these components of discriminability. Indeed, Eriksen \& Wechsler (1955) observed that in a task involving judgments of sizes of squares, arousal (anxiety) created by electric shock produced no difference in overall discriminability ( $T x y)$, compared to a nonshock condition, but that the arousal did result in a decrement in both response variability $(\mathrm{Hy})$ and response unreliability (Hxy).

The present research extends the analysis of this situation by taking into account two factors in addition to type of arousal. This first is task difficulty. Because a more difficult discrimination task could lead to greater intrusion of competing responses, shock or noise could have a greater effect than in a less difficult task. Second, because Ss' level of personal anxiety might augment arousal effects (Spence \& Spence, 1966), such dispositional differences, as measured by the Manifest Anxiety Scale (MAS) of Taylor (1953), were incorporated in the two studies presented below.

\section{METHOD}

In both studies the design was a 2 by 2 by 2 factorial consisting of two levels of stimulus difficulty, two levels of arousal, and two levels of manifest anxiety. In Study 1, 48 Ss (approximately half were male and half female) were assigned to experimental conditions on the basis of having MAS scores above or below the median in the larger undergraduate class from which they were drawn. Arousal was created by means of a series of 16 short bursts of white noise introduced for varying ( $1-2 \mathrm{sec})$ durations through earphones during randomly selected intervals between stimulus presentations. In the No-Arousal condition, Ss received no white noise and did not wear earphones. Verbal report and observations of Ss receiving white noise attested to the effectiveness of the arousal stimulus in terms of greater feelings of temotional disturbance and irritability in the Arousal group than in the No-Arousal group. Task difficulty was varied by using one of two series of 11 line lengths projected onto a screen in a semi-darkened room. In the Hard condition, the range of lengths was from $1 \frac{1 / 2}{2}$ in. to $4 \mathrm{in}$., in increments of $1 / 4 \mathrm{in}$. In the Easy condition, the lengths ranged from $1 / 2$ in. to 8 in. in $3 / 4$-in. increments. Ss were instructed to call out a response after each stimulus presentation, using an 11-category numerical response such that 1 was the shortest stimulus and 11 was the longest. Each stimulus was presented in blocks of seven trials with a 5-sec interval between each of the 77 stimulus presentations.

A similar procedure was followed in Study 2, in which 80 undergraduate $S s$ were assigned to one of two stimulus conditions (Hard vs Easy). The same stimuli were used as in Study 1, except that they were presented nine times. As in Study 1 , Ss above and below the median on the MAS constituted the two levels of anxiety. Electric shock was the arousal stimulus in Study 2. In the Arousal condition, Ss received a just tolerable level of 12 brief bursts of electric current at random intervals between presentations of the 99 stimuli. Ss in the No-Arousal condition received no shock and were not connected to the shock apparatus.

\section{RESULTS OF STUDY}

Separate analyses of the Txy, Hy, and Hxy measures were carried out. For Txy scores, significant main effects were found for Arousal vs No-Arousal $(F=11.40$, df $=1 / 40$, $p<.001)$ and for Hard vs Easy stimulus series $(F=139.38$. $\mathrm{df}=1 / 40, \mathrm{p}<.001)$. Differences in discriminability as a function of MAS scores were not found to be significant, nor were any of the interactions significant. Analysis of group means indicates the Easy stimuli were discriminated significantly better than the Hard stimuli (mean Txy $=2.30$ and 1.52 , respectively). Further, Ss receiving white noise had lower discriminability scores than those not receiving white noise (mean $\mathrm{Txy}=1.84$ and 2.03, respectively). Analysis of the response variability measure, Hy, indicated that of the three main effects, only the Hard. vs Easy stimulus difference was significant $(\mathrm{F}=31.38, \mathrm{df}=1 / 40, \mathrm{p}<.001)$, such that the Easy series resulted in more response variability than did the Hard series (mean $\mathrm{Hy}=3.26$ and 2.96 , respectively). Analysis of $\mathrm{Hxy}$ also indicated a main effect of Hard vs Easy stimuli $(F=27.32, \mathrm{df}=1 / 40, \mathrm{p}<.001)$ such that Hard stimuli were responded to more unreliably than were Easy stimuli (mean $\mathrm{Hxy}=4.86$ and 4.42 , respectively). In addition, a significant main effect of Arousal vs No-Arousal was observed $(F=6.81$, $\mathrm{df}=1 / 40, \mathrm{p}<.05)$, such that $\mathrm{Ss}$ receiving noise were more unreliable than were $S$ s receiving no noise (mean $H x y=4.72$ and 4.55 , respectively).

Table 1 presents the mean scores for Txy. Hy, and Hxy as a function of the arousal and stimulus difficulty variables. It will be noted in Table 1 that for both Hard and Easy stimuli, the effect of arousal was to reduce discriminability, and that this

Table 1

Study 1: Mean Txy, Hy, and Hxy Scores as a Function of Arousal and Difficulty Level of Stimuli

\begin{tabular}{llll}
\hline \multicolumn{1}{r}{ Conditions } & Txy & Hy & Hxy \\
\hline No Arousal-Easy & 2.37 & 3.31 & 4.40 \\
Arousal-Easy & 2.23 & 3.21 & 4.44 \\
No Arousal-Hard & 1.69 & 2.93 & 4.70 \\
Arousal-Hard & 1.45 & 3.00 & 5.01 \\
\hline
\end{tabular}


Table 2

Study 2: Mean Txy, Hy, and Hxy Scores as a Function of Arousal and Difficulty Level of Stimuli

\begin{tabular}{llll}
\hline \multicolumn{1}{c}{ Conditions } & Txy & Hy & Hxy \\
\hline No Arousal-Easy & 2.34 & 3.35 & 4.50 \\
Arousal-Easy & 2.31 & 3.31 & 4.43 \\
No Arousal-Hard & 1.65 & 3.08 & 4.89 \\
Arousal-Hard & 1.69 & 3.07 & 4.84 \\
\hline
\end{tabular}

difference was larger for the Hard series. Inspection of the mean Hy and Hxy scores in Table 1 suggests the basis of the poorer discriminability under conditions of arousal. It will be noted that the effect of arousal on $\mathrm{Hy}$ was opposite in each stimulus condition, such that response variability decreased under arousal on the Easy series while it increased under arousal on the Hard series. These results alone would suggest that arousal would increase discriminability in the Hard stimulus condition. However, inspection of the Hxy means in Table 1 indicates why this is not the case. That is, in both stimulus conditions the effect of arousal is to increase response unreliability, but this increase is much greater in relation to the Hard stimulus condition. One index of this greater unreliability of the Arousal-Hard group compared to the No Arousal-Hard group is the observation that 11 of the $12 \mathrm{Ss}$ in the former group assigned the same response at least once to six or more different stimuli, while only five of $12 \mathrm{Ss}$ had this characteristic in the latter group $(p=.025$, Fisher-Yates exact probability test).

\section{RESULTS OF STUDY 2}

Analysis indicated that of the three main variables, only the Hard-Easy stimulus difference had a significant effect on the three information measures. In relation to discriminability, the Ss in the Hard stimulus condition had a mean Txy score of 2.32, while those in the Easy stimulus condition had a mean Txy value of $1.67(\mathrm{~F}=140, \mathrm{df}=1 / 72, \mathrm{p}<.001)$. In terms of response variability, the Easy stimulus condition yielded an $\mathrm{Hy}$ mean of 3.33 , while the Hard stimulus condition y ielded a mean $\mathrm{Hy}$ of $3.08(\mathrm{~F}=21.08, \mathrm{df}=1 / 72, \mathrm{p}<.001)$. In relation to the response unreliability measure, those $S s$ in the Hard stimulus condition had a mean $\mathrm{Hxy}$ value of 4.87 while those in the Easy stimulus condition had a mean $\mathrm{Hxy}$ value of 4.46 ( $F=39.30 . \mathrm{df}=1 / 40, p<.001$ ). Inspection of means of these information measures as a function of arousal and stimulus difficulty indicates areas of difference in contrast to Study 1.

As in Study 1. the effect of arousal with Easy stimuli was to produce a slight decrease in both Txy and Hy. However, in the case of Hxy in Study 2, an increase in reliability (decreased Hxy) is observed, both for the Easy and the Hard stimulus series. Thus, response variability (Hy) is almost unchanged for Hard stimuli with the arousal of shock, and this combined with the increased reliability with shock contributes to the overall increase in Txy for the Hard-Arousal condition.

\section{DISCUSSION}

The results of these two studies indicate that while aversive arousal may have a debilitating effect on discriminability, the basis for this negative effect may differ as a function of the specific form of arousal. In the case of electric shock, both the results of Study 2 and of Eriksen and Wechsler suggest that response unreliability decreases with shock, Ss tending to respond in a more stereotyped manner. In part, at least, such biasing in responding would appear to be an effect of anxiety as drive increasing the tendency to use some responses more than others (Brody, 1964; Kuethe \& Eriksen, 1957). The effect of white noise in significantly increasing response unreliability would suggest that this arousal stimulus serves less to reduce discriminability through drive properties and more through a distractibility effect. The net effect of such a loss of attention is to increase the random character of responding in a discrimination task, particularly when the stimuli are more difficult to discriminate, such that any gain in overall response variability ( $\mathrm{Hy})$ is vitiated by the substantial increase in response unreliability (Hxy). These results underscore the need for caution in generalizing about the effects of aversive arousal states upon discrimination behavior. REFERENCES

BIERI, J., ATKINS, A. L., BRIAR, S., LEAMAN, R. L., MILLER, H. \& TRIPODI, T. Clinical and social judgment: The discrimination of behavioral information. New York: Wiley, 1966.

BRODY, N. Anxiety, induced muscular tension, and the statistical structure of binary response sequence. Journal of A bnormal and Social Psychology, 1964, 68, 640-643.

ERIKSEN, C. W., \& WECHSLER, H. Some effects of experimentally induced anxiety upon discrimination behavior. Journal of Abnormal and Social Psychology, 1955, 51, 458-463.

GARNER, W. R. Uncertainty and sinucture as psychological concepts. New York: Wiley, 1962.

KUETHE, J. L., \& ERIKSEN, C. W. Personality, anxiety, and muscle tension as determinants of response stereotypy. Journal of Abnormal and Social Psychology, 1957, 54, 400-404.

SPENCE, J. T., \& SPENCE, K. W. The motivational components of manifest anxiety: Drive and drive stimuli. In C. D. Spielberger (Ed.), Anxiety and behavior. New York: Academic Press, 1966.

TAYLOR, J. A. A personality scale of manifest anxiety. Joumal of Abnormal and Social Psychology, 1953, 48, 285-290. NOTE

1. This research was supported by Grant GS-842 from the National Science Foundation. 